\title{
Photo-induced Chiral Switching of a Stilbene-Binaphtyl Supramolecular System
}

\author{
Takenori Tose ${ }^{1,3}$ and Takashi Yamashita*1,2 \\ ${ }^{1}$ Department of Pure and Applied Chemistry, Tokyo University of Science, \\ 2641 Yamazaki, Noda, Chiba 270-8560, JAPAN \\ ${ }^{2}$ School of Computer Science, Tokyo University of Technology, \\ 1404-1 Katakura, Hachioji, Tokyo 192-0982, JAPAN \\ ${ }^{3}$ RIKEN, 2-1 Hirosawa, Wako, Saitama 351-0198 JAPAN \\ e-mail:17214660@ed.tus.ac.jp, ${ }^{2}$ yamashitatks@stf.teu.ac.jp
}

Keywords: Chiral Switch, Stilbene, Photo reaction, Supramolecule

\section{Introduction}

Photochemical reactions are one of the tools to control molecular structure and properties, as well as those of various supramolecules such as, rotaxanes and catenanes which can be manipulated by photochemical stimuli. These molecules are expected to be applied to molecular switches, optical memory, photo-mechanical materials, logic gates and so on [1].

We have reported novel light-driven chiral compounds containing azobenzene moiety as a photo responsive group and binaphthyl moiety as a chiral source in a single molecule, to have found that the chirality of the molecule can be reversibly switched by photoirradiation, because trans-cis photo-isomerization of azobenzene moiety changes the face angle of the binaphthyl group [2]. Cis-trans thermal backward isomerization of azobenzene moiety took place even at room temperature in the molecular system, however, resulting in the loss of the stored chiral information. In this paper we synthesized novel photo responsive chiral cyclic molecule containing stilbene instead of azobenzene moiety to increase the thermal stability, and then we evaluated the photo response and the chiral optical properties of the material [3].

\section{Experimental}

\subsection{Material}

4SES was synthesized by Williamson reaction of (S)-(-)-1,1'-bi-2-naphthol and 1,2dibromoethane-capped dihydroxy stilbene which was prepared by McMurry coupling of 4-hydroxybenzaldehyde (Fig. 1).

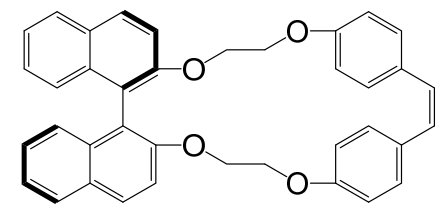

Fig.1 chemical structure of 4SES used in this study.

\subsection{Measurement}

Photo irradiation of 4SES in acetonitrile was performed by $254 \mathrm{~nm}$ light with a low pressure mercury lamp and by $365 \mathrm{~nm}$ light with an ultrahigh pressure mercury lamp. Thermal isomerization of 4SES was measured in acetonitrile or toluene, which was heated up to 338 $\mathrm{K}$ and $373 \mathrm{~K}$.

\section{Results and Discussion}

Photo-irradiation of 4SES in acetonitrile at $254 \mathrm{~nm}\left(250 \mu \mathrm{W} / \mathrm{cm}^{2}, 60 \mathrm{sec}\right)$ induced cis-trans isomerization of the stilbene moiety with increase in absorption at $330 \mathrm{~nm}$ and decrease at $280 \mathrm{~nm}$. On the other hand, Photo-irradiation at $365 \mathrm{~nm}$ $\left(180 \mu \mathrm{W} / \mathrm{cm}^{2}, 60 \mathrm{sec}\right)$ reproduced cis-isomer by reversible trans-cis isomerization with showing isosbestic point at $297 \mathrm{~nm}$ (Fig. 2). 


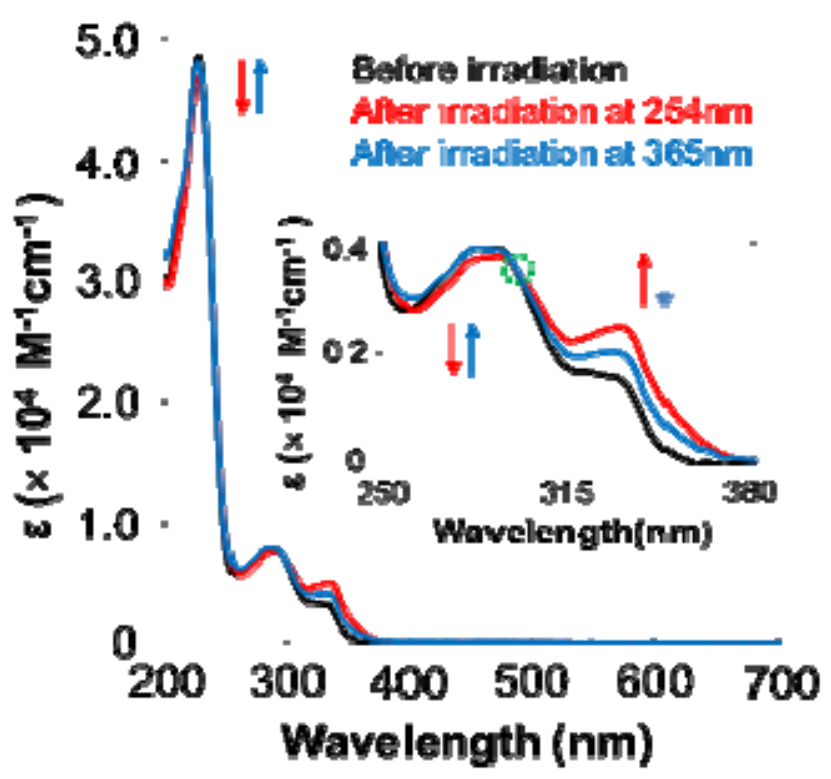

Fig.2 UV-Vis spectra of 4SES in acetonitrile solution (black), after photoirradiation at $254 \mathrm{~nm}$ (red), and after photoirradiation at $365 \mathrm{~nm}$ (blue).

Fig 3 shows change in CD spectrum of 4SES in acetonitrile under photoirradiation. Circular dichroism $(\Delta \varepsilon)$ of 4 SES decreased at $230 \mathrm{~nm}$ and $250 \mathrm{~nm}$ by the photoirradiation at $254 \mathrm{~nm}$, while the CD signal reversibly changed by photoirradiation at $365 \mathrm{~nm}$, showing that the circular dichroism of binaphthyl moiety was induced by the isomerization of stilbene moiety in 4SES (Fig.3).
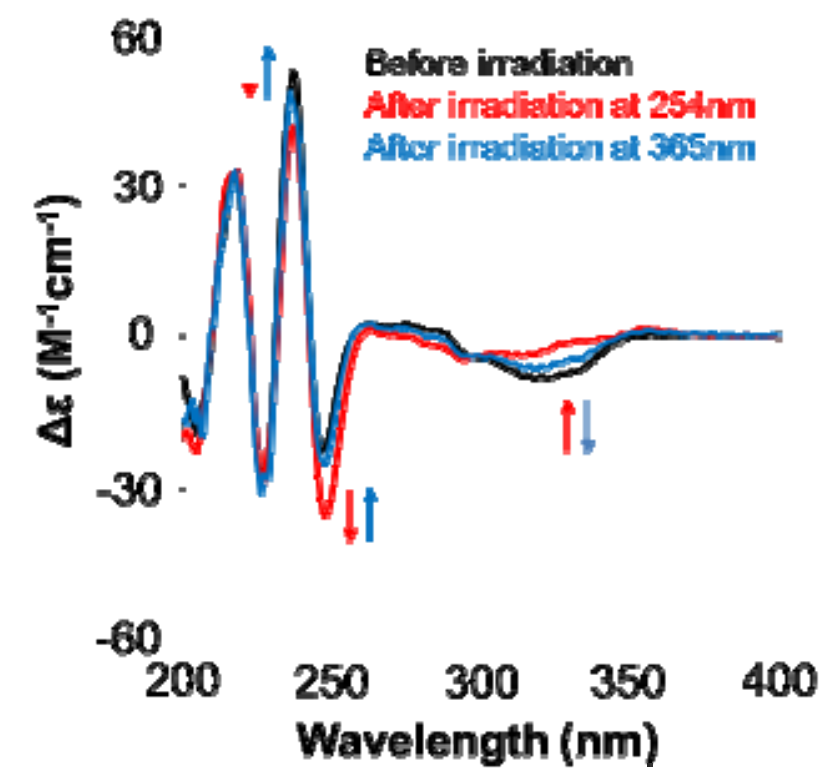

Fig. 3 CD spectra of 4SES in acetonitrile (black), after irradiation (red) at $254 \mathrm{~nm}$, and after irradiation (blue) at $365 \mathrm{~nm}$.

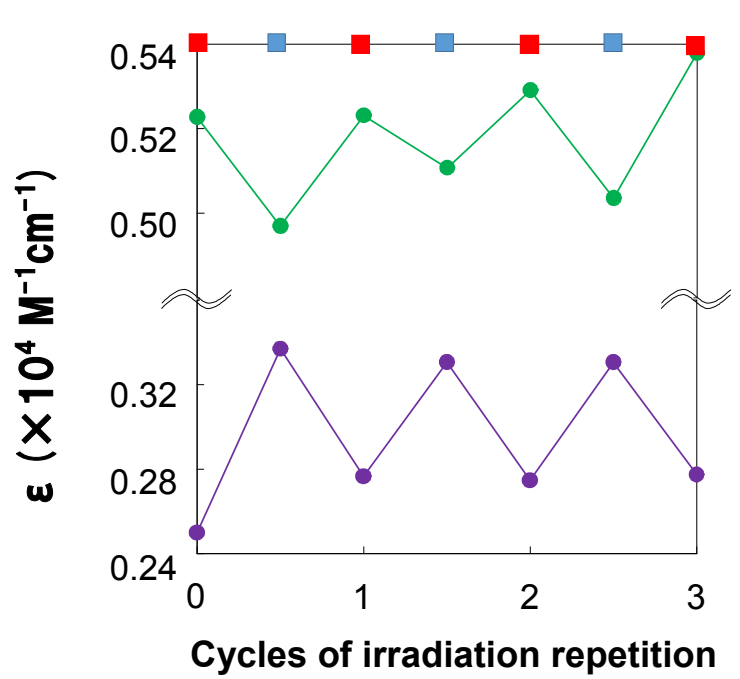

Fig. 4 Circular dichroism change during repetition cycle of 4SES at $280 \mathrm{~nm}$ (purple line) and $330 \mathrm{~nm}$ (green line) after photoirradiation at $254 \mathrm{~nm}$ (red region), and photoirradiation at $365 \mathrm{~nm}$ (blue region).

The CD signal change is reversibly reproduced by the photoirradiation at $254 \mathrm{~nm}$ and $365 \mathrm{~nm}$, corresponding to the photoisomerization of stilbene moiety, shown in Fig. 4.

In order to estimate the thermal stability of cis-form of 4SES, an acetonitrile solution of 4SES was prepared, and then the spectral change was compared between trans-SES, cis-4SES, and cis-3RAA, heating up to $338 \mathrm{~K}$. Absorption of cis-3RAA rapidly decreased with rate constant of $3.3 \times 10^{6} \mathrm{~s}^{-1}$ by cis-trans thermal back isomerization of stilbene moiety, while no change in absorption was observed for trans-4SES nor cis-4SES after heating for 2 hours (Fig 5). The difference is based on the thermal stability of cis form of stilbenes compared to that of azobenzene derivatives. In order to confirm the stability of the cis-4SES, absorption change of cis-4SES was measured at elevated temperature. Fig 6 shows the change in absorption of cis-4SES in toluene solution heated up to $100{ }^{\circ} \mathrm{C}$ for 2 hours, but no thermal backward reaction was observed at all.

\section{Conclusion}

Novel photoresponsible chiral supramolecule 4SES was prepared and its chiral switching and thermal stability were measured. 4SES shows reversible $C D$ change up on photoirradiation, due to face angle change of binaphthyl group induced by trans-cis photoiromerization. The cis isomer of 

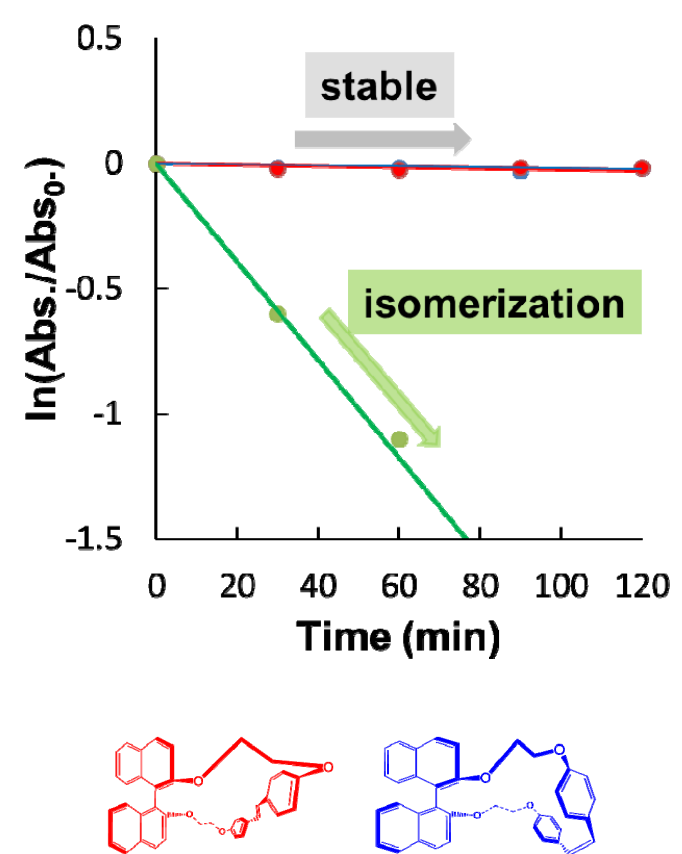

trans-4SES

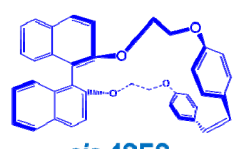

cis-4SES

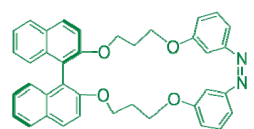

cis-3RAA

Fig.5 Plots of $\ln \left(\mathrm{Abs} / \mathrm{Abs}_{0}\right.$. $)$ of trans-4SES, cis-4SES, and cis-3RAA in $\mathrm{CH}_{3} \mathrm{CN}$ at $338 \mathrm{~K}$ [2].

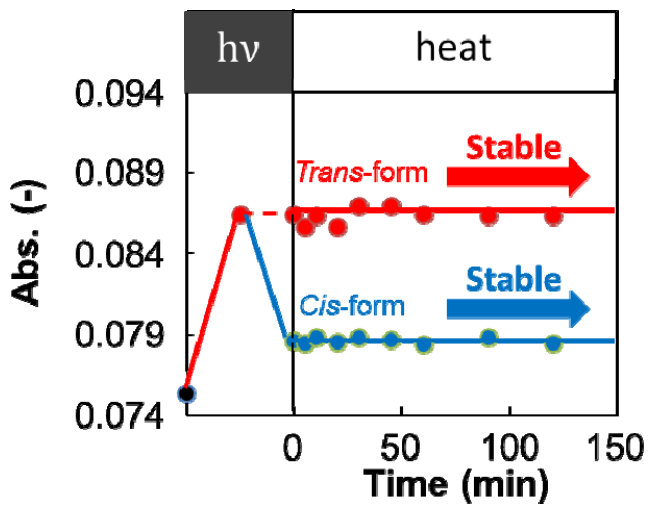

Fig.6 Absorption change of 4SES in toluene after photoirradiation to convert cis form, followed by heating at $373 \mathrm{~K}$.

4SES has high thermal stability whose absorption does not change even by heating at $100{ }^{\circ} \mathrm{C}$ for 2 hours, which is quite contrast to the case of 3RAA. 4SES is expected as the potential materials for chiral optical data storage, asymmetric synthesis, and so on.

\section{Reference}

1. Murakami, H.; Kawabuchi, A. Matsumoto, R.; Ido, T.; Nakashima, N. J. Am. Chem. Soc. 127 (2005) 15891.

2. M. Kawamoto, N. Shiga, K. Takaishi, and T. Yamashita, Chem. Comm., 44 (46) (2010) 8346-8349.

3. Michael M. Pollard, Auke Meetsma, Ben L. Feringa, Org. Biomol. Chem., 6 (2008) 507. 